\title{
Automated Systems in the Aviation and Aerospace Industries
}

Tetiana Shmelova

National Aviation University, Ukraine

Yuliya Sikirda

Kirovograd Flight Academy of the National Aviation University, Ukraine

Nina Rizun

Gdansk University of Technology, Poland

Dmytro Kucherov

National Aviation University, Ukraine

Konstantin Dergachov

National Aerospace University - Kharkiv Aviation Institute, Ukraine 
Published in the United States of America by

IGI Global

Engineering Science Reference (an imprint of IGI Global)

701 E. Chocolate Avenue

Hershey PA, USA 17033

Tel: 717-533-8845

Fax: 717-533-8661

E-mail: cust@igi-global.com

Web site: http://www.igi-global.com

Copyright (C) 2019 by IGI Global. All rights reserved. No part of this publication may be reproduced, stored or distributed in any form or by any means, electronic or mechanical, including photocopying, without written permission from the publisher. Product or company names used in this set are for identification purposes only. Inclusion of the names of the products or companies does not indicate a claim of ownership by IGI Global of the trademark or registered trademark.

Library of Congress Cataloging-in-Publication Data

Names: Shmelova, Tetiana, 1961- editor.

Title: Automated systems in the aviation and aerospace industries / Tetiana

Shmelova, Yuliya Sikirda, Nina Rizun, Dmytro Kucherov, and Konstantin

Dergachov, editors.

Description: Hershey, PA : Engineering Science Reference, [2019] I Includes

bibliographical references.

Identifiers: LCCN 2018036333| ISBN 9781522577096 (h/c) | ISBN 9781522577102

(eISBN)

Subjects: LCSH: Aeronautics--Technological innovations. I Aerospace

industries--Automation. I Automatic pilot (Airplanes) | Aeronautics--Study

and teaching--Data processing.

Classification: LCC TL553 .A847 2019 I DDC 629.1--dc23 LC record available at https://lccn.loc.gov/2018036333

This book is published in the IGI Global book series Advances in Mechatronics and Mechanical Engineering (AMME)

(ISSN: 2328-8205; eISSN: 2328-823X)

British Cataloguing in Publication Data

A Cataloguing in Publication record for this book is available from the British Library.

All work contributed to this book is new, previously-unpublished material. The views expressed in this book are those of the authors, but not necessarily of the publisher.

For electronic access to this publication, please contact: eresources@igi-global.com. 


\title{
Chapter 7 Synthesis of the Laws of Motion Control of a UAV Group With Natural Obstacles
}

\author{
Dmytro Kucherov \\ National Aviation University, Ukraine \\ Minglei Fu \\ Zhejiang University of Technology, China \\ Andrei Kozub \\ National University of Defense of Ukraine, Ukraine
}

\begin{abstract}
A solution is proposed for the task of controlling a group of UAVs moving along a given route. The group is considered as a limited-size formation consisting of n-agents moving relative to the leader, which allows us to treat the group as some aggregate with the center of motion. The quantitative composition of a group can change while maintaining the integrity of the group. The chapter proposes the use of smooth laws governing the motion of a group. The safety of motion is ensured by introducing into the law of control the components equivalent to the creation of attractive and repulsive fields.
\end{abstract}

\section{INTRODUCTION}

Group or collective management is one of the priority areas for the development of technical systems. This is due to an increase in the number of technical objects endowed with a certain share of artificial intelligence, which in turn requires the resolution of technical issues related to the organization of their joint actions, such as coherence, mutual assistance, and security.

The first among them is consistency, which is manifested in synchronous movements with an observance of distances, intervals, and exceedances when considering these objects in 3D space. This requires traffic control, for example, when driving along a route. For a UAV of various configurations, en-route movement is a task of varying complexity, for example, when flying in free space where there 
are no obstacles, at low and very low altitudes, where obstacles are surface structures, or, for example, in confined spaces need different maneuverability characteristics are required.

Complex maneuvers are capable of performing UAVs of special designs. A good maneuvering is distinguished quadrotors - 4-engine aircraft. They are able to hang over the subject and sharply change the direction of motion. Currently, most of the technical issues associated with ensuring their flight, namely: power supply, the execution of hulls, hangers are to some extent resolved. Nevertheless, the development and programming of controllers that implement effective control, and especially the implementation of a group or swarm control remains an actual task.

Advantages of collective management lie in the possibility of obtaining better characteristics of time, accuracy, the probability of accomplishing the final task assigned to a group of air platforms. Recently, a group of objects that provide a joint task is called a multi-agent system, a separate element of the system is an agent capable of performing a number of the simplest activities. The actions of the system's agents must be safe for other agents. In this case, the group should be given an allowable area within which the actions of an agent do not interfere with other agents of the system. From the experience of group flights of manned air vehicles, there are known minimum distances equivalent to $2-3$ hulls of the vehicles and ensuring safe maneuvering.

Existing approaches to the solution of the problem of swarm management are borrowed from observations of the behavior of bio-systems such as an anthill, a swarm of bees, a flock of birds. To achieve such mutual understanding and coordination of movements what have this listed bio-systems, it is necessary to develop new algorithms, which this section is devoted to. The proposed algorithms are based on modern achievements of science in the field of control of UAV, and also offers a number of solutions for traditional tasks of traffic control along a given route, and also considers possible reconstructions that are of interest for the study of movements in a confined space.

It is believed that a useful task performed by a quadrotor group is monitoring the earth's surface. To accomplish this task, the robotics platform is equipped with a target and auxiliary equipment that provides the acquisition and transfer of information to the point of reception and analysis. Therefore, the main efforts are directed to the development and synthesis of control laws that ensure the action of the UAV agent under standard flight conditions, and also the properties of control laws that ensure a stable and controlled movement whole the group are established.

The section is structured as follows: the next subsection contains an overview of the works that allows us to evaluate the state of the problem. The problem statement and the description of the UAV group are proposed in Section 3. In Section 4 the analysis of the stability of various types of movements is given. The procedure for synthesizing the control law is presented in Section 5. Simulation of the movement of a group's element and the group as a whole is given in Section 6. Conclusions on the work are presented in the last section.

\section{RELATED WORKS}

There are many studies on multi-agent systems. The most general idea and concepts of multi-agent systems are given in fundamental publications (Hoek \& Wooldridge, $2008 \&$ Murray, 2007). Fundamental research in this area is devoted to the formalization of knowledge representations, for example, as is done by (Hoek \& Wooldridge, 2008). Two areas are of interest here. The methods of representing the intelligent state of agents and the methods for determining the rational actions that lead to this state are referred to 
the first direction. The second direction is to describe the structure of the multi-agent environment and to provide the authorities that agents are assigned to act as part of the group.

The key applications of group control systems and the description of algorithms for their implementation are presented in the papers (Murray, 2007; Shmelova et al, 2016). The areas covered include the use of unmanned vehicles and aircraft, as well as mobile sensor networks. Each control problem consists of a description of the system and their final position by a system of first-order differential equations, and also by the definition of an integral function of quality (productivity) that represents a certain amount of cost, in particular, it can be time and a final state. Control law in the control problems under consideration is a smooth function of the state and the role of the machine.

Many works in this area are aimed at studying the consensus problem in the implementation of joint actions. Among them related this works (Ren et al 2005; Rezaee \& Abdollah, 2016; Olfati-Saber et al, 2007; Amelin et al, 2013; Ni \& Cheng, 2010; Ma \& Zhang, 2010). Thus, the authors (Ren et al 2005) carried out an analysis of the coordination of actions of the multi-agent system to achieve coordinated actions. Consistency is understood as the correspondence of information states of interacting agents. For this, the agents of the system are provided with coordinating information. The conditions for achieving consensus in the conditions of a constancy of topology in the exchange of information and under conditions of dynamically changing topology are determined. The authors note that the analysis is convenient if the process of information exchange is described by integrators not higher than the second order.

In (Ren et al, 2005), a protocol was obtained for coordinating the actions of agents in a multi-agent linear system consisting of underwater autonomous machines having a randomly switched topology. The problem is solved with the help of supermartingales and solving linear matrix inequalities. The problem solution does not depend on the topology of the system and is not computationally expensive.

An overview of the key concepts of the theory of consistency and its applications for network systems within a single structure is presented in (Rezaee \& Abdollah, 2016). The work includes basic concepts of consistency in information theory and methods of control of convergence, as well as analysis of the effectiveness of reconciliation protocols based on theories of matrix analysis and spectral graphs. The network system is built on the basic assumption of a local interaction of mobile or static agents, equipped with means of measuring the state, performing calculations and communication. An analysis of the behavior of the flock is shown, and new algorithms and principles for the synthesis of fault-tolerant individual nodes and channels of sensor networks are developed.

Optimization of the flight of small UAVs based on the consensus approach is considered in (OlfatiSaber et al, 2007). To control flight, thermal ascending flows are used. The center of thermal ascending fluxes is detected by the stochastic approximation algorithm. The use of thermal ascending flows saves energy during the flight.

The consensual problem of multi-level high-order agent systems is considered in (Amelin et al, 2013). In the system, the dynamics of each agent and the leader is a linear system. The management of each agent is constructed using local information, as well as a detailed analysis of the consensus on leadership for different topologies, obtained through the description of agents as a linear system, which makes it possible to synthesize the management of linear algebra.

Coherence is a fundamental problem in the field of MAS research. The necessary condition for consistency with respect to a set of admissible consensus protocols for multi-agent systems (MAS) is studied in (Ni \& Cheng, 2010) on the basis of the joint influence of a dynamic agent with a linear structure and the coupling topology on consistency. For MAS with fixed topology and stationary agents, necessary and sufficient conditions are shown. 
In (Ma \& Zhang, 2010) discusses the consensual problem of managing multiple agents with a delay in communication time. Here the dynamics of each agent is described by a system of linear differential equations of the second order, the time delay of the connection is not constant and the interconnections of agents are represented by a directed diagram. The paper also proposes a necessary and sufficient condition for the stability of control for continuous and switching systems.

In (Hu \& Hong, 2007; Zhang \& Chen, 2014; Ferber et al, 2004; Xiao et al, 2009; Franchi et al, 2011; Pipattanasomporn et all, 2011; Kala \& Warwick, 2012; Hao et al, 2018; Flemisch et al, 2017; Olfati-Saber et al, 2003; Koo \& Shahruz, 2001), the results of the optimization of multiple-agent systems by different methods are presented. In (Hu \& Hong, 2007), the solution is proposed to be a linear multi-agent system based on the construction of the feedback vector in the system. The feedback vector is constructed on the basis of the solution of a linearly-quadratic problem. The authors also show that, in order to achieve a two-sided consensus, a pair of factors are crucial, one of which is the appropriate choice of estimating a consensus error, and the other is an appropriate topology of the graph that requires the existence of a spanning tree and the structural balance of the digraph.

In (Zhang \& Chen, 2014), an analysis is proposed for the effectiveness of multi-agent systems based on the minimal description of the model in the form of agent-group-role (AGR). This approach involves eliminating the drawbacks of the model proposed by the MAS community. Among them is the openness of the agent, which causes security problems, modularity, which makes the structure cumbersome in upgrades and excludes the possibility of flexible integration of substructures, as well as the need for an identical structure of agents based on the unification of the programming language, which causes interaction problems between agents.

The main idea of the authors of the article (Ferber et al, 2004) assumes the separation of information in the multi-agent system into global and local. At the same time, global information is provided to the leader and agents responsible for the navigation of the team and also determines the geometry of the desired formation. Local information determines the relative positions of followers in the framework of the education established by leaders. This type of information is only available during the interaction process. The leader is determined by the topology of the information flow, and not by the type of information. The proposed approach allows us to define a new class of nonlinear consensus protocols for coordinating the state of multi-agent systems. The protocols considered have the form of continuous feedback with a finite convergence time, which can be useful for multi-agent systems with many agents.

In the paper (Xiao et al, 2009) proposes a distributed control strategy for remote control of a fleet of unmanned aerial vehicles (UAVs). Control methods are considered that provide flexible UAV behavior for arbitrary separation and integration, ensuring system stability. The general remote control system is considered passive and has stable behavior both in free movement and in interaction with unknown passive obstacles.

Intellectual control of a multi-agent system describing a distributed power circuit is considered in (Franchi et al, 2011). Agents in the multi-agent system work together to detect upstream faults and react accordingly, providing autonomous operation of the microgrid. This capability can be perceived as an alternative to the software of a traditional zone protection system that isolates microgrid. Multi-agent system provides more flexible protection, redefining the zone boundary "on the fly."

Planning the movement of mobile ground robots through the introduction of lateral potentials as measures that allow vehicles to solve their lateral positions on the road is proposed in (Pipattanasomporn et all, 2011). 
The authors of (Kala \& Warwick, 2012) proposed an optimization strategy for a game with overloads when the objects of the system are representable in a matrix form. A necessary and sufficient condition is formulated to ensure the conversion of the system into a game with overload according to a given performance criterion of the system presented in the form of a potential function based on the development of the proper functions of the value of the object. Using this technology allows you to achieve a global optimization of the dynamic system based on objects in which each agent optimizes its cost function. This approach can be extended to those systems that are partially or almost convertible. The proposed methodology can be applied only to classic games with overloads, where a user with several unit requirements is not allowed.

In (Hao et al, 2018) technologies of group management of aviation and automobile vehicles in automated control systems are presented. The authors note technological and ethical reasons limiting the possibility of realizing a fully autonomous vehicle in the near future. The paper outlines the principles of control based on concepts of separate and cooperative management, as well as the concept of people's control and cooperation and an unmanned vehicle.

Algebraic representation of formations on the basis of triangulated graphs enabled the authors (Flemisch et al, 2017) to determine the cost of the task by a group of vehicles. This presentation together with navigation information allowed the authors to formally solve the problem of tracking the desired trajectory behind a group of six cars and optimizing their actions.

The laws governing the group of unmanned aerial vehicles (UAV) relative to the leader of the group that specifies the required trajectory of flight of driven vehicles is presented in (Olfati-Saber et al, 2003). The paper proposes a solution to the control problem for separate and simultaneous take-off scenarios.

A description of the interaction of the agents by the Laplacian matrix (simply Laplacian) or a pair of independent digraphs (by two Laplacian matrices) is presented in (Koo \& Shahruz, 2001), where the estimates of the largest lower bound of the bounded Rayleigh coefficient with respect to the general OC vector and the Laplace EB matrix characterizing the convergence of the agents to the leader.

The practical application of multi-agent systems is given in $(\mathrm{Li}, 2018$; Filaretov et al, 2017; Yu et al, 2017; Baolin et al, 2017; Ögren et al, 2004; Liu et al, 2017; Han et al, 2017; Yun \& Jian, 2018; Xie et al, 2018). In (Li, 2018), the development of a joint control system for a group of mobile robots (MR) based on the onboard, universal information management system (ICS) is considered. To implement this cooperative management system, it is proposed to introduce an additional component that ensures the interaction of MR during their transfer to the ICS of each MR. The leader-follower strategy is used to consider the data bandwidth limitation between the MR group. The conducted studies confirm the high efficiency of the proposed system.

The authors of (Filaretov et al, 2017) consider a multi-agent system in the form of a platoon of vehicles following the main line. The law of management is based on the principles of feedback and takes into account the road bias. The individual stability of the vehicle is estimated by the Raus-Hurwitz method.

Replacing the traditional mechanism of deploying a dynamic object with two degrees of freedom by an object with many degrees of freedom similar to the skeleton mechanism of Chrysopelea is proposed in (Yu et al, 2017). A dynamic structure consisting of a combination of serial and parallel connections driven by servo motors is described by some multi-agent matrix. The effectiveness of managing this structure is achieved by introducing an algorithm for optimizing system parameters based on swarm optimization.

The strategy of managing groups of vehicles in moving and reconfiguring in response to a distributed medium response is shown in (Baolin et al, 2017). Each vehicle in the group serves as a mobile sensor 
and the transport network is a matrix of sensors capable of moving and changing the configuration. The proposed management strategy divides the management of network formation from performing network maneuvers. To achieve the management effect, a coordination structure has been developed that uses virtual bodies and artificial potentials, which allows vehicles to avoid obstacles on the route. The task of searching for a mobile network of sensors with a local maximum in the field of the environment is being constructed. The network can adapt its configuration in response to a sensitive environment in order to optimize its gradient ascent.

In (Ögren et al, 2004), the problem of distributed cooperative management under conditions of reconfiguration of a UAV swarm was investigated. The consensus protocol has a linear form of the law of feedback, sufficient conditions for achieving the required reconfiguration of the UAV formation are obtained on the basis of the Lyapunov theory and the LMI method.

The reconfigurable wireless control system (Liu et al, 2017) is designed for a robotic system with two hands interacting with each other. It appears to be a distributed multi-layer system consisting of a coordinated controller, two motion controllers corresponding to two arms, and ZigBee wireless communication modules.

Development of an intelligent towing system for water transport, where manual interaction has been preserved so far, is presented in (Han, 2017).

The control system for the position and height of a group of space vehicles is presented in (Yun \& Jian, 2018). The proposed control system uses the algorithm of adaptive cooperative control, in which the estimates of the coordinates of the spacecraft in the presence of measurement interference are estimated by the method of extended least ones for several spans.

A review of co-management with fixed time in multi-agent systems, ensuring a guaranteed time of establishment, regardless of the initial conditions, is presented in (Xie et al, 2017). These algorithms are able to provide better characteristics of closed overlap and deviation from interference in comparison with asymptotic control algorithms. Unlike finite-time control, co-management with fixed time provides a higher convergence rate and provides a clear estimate of the establishment time, regardless of the initial conditions, which is desirable for multi-agent systems.

In papers (Zuo et al, 2018; Kucherov et al, 2016; 2014; 2015; Kulik \& Dergachov, 2016; Kozlovskyy, 2016), problems associated with the environment of systems of a similar kind are considered. The main problem of this chapter is the methodology for synthesizing the management of a multi-agent system when it moves along a certain route, where obstacles are possible, the overcoming of which is not associated with a height maneuver.

\section{PROBLEM STATEMENT}

The actions of the UAV group performing the task of airspace monitoring are considered. We denote a separate UAV that is part of the group by the object $A_{i}$. We introduce the Euclidean space $X Y Z$ to describe the current position of Ai in space. Let consider an arbitrary motion of $S_{i}$ objects in the chosen space. In the interests of the task, the objects start, assemble into a group, perform a group flight, perform some flight mission, and then complete the flight at a given point. The flight mission in question is shown in Figure 1, which is performed by a group of 4 UAVs.

Figure 1 shows the route of the group, on this route, there are obstacles of natural origin or single buildings. When determining the mission, the presence of obstacles was not taken into account. It is 
Figure 1. Group of UAVs, $N=4$

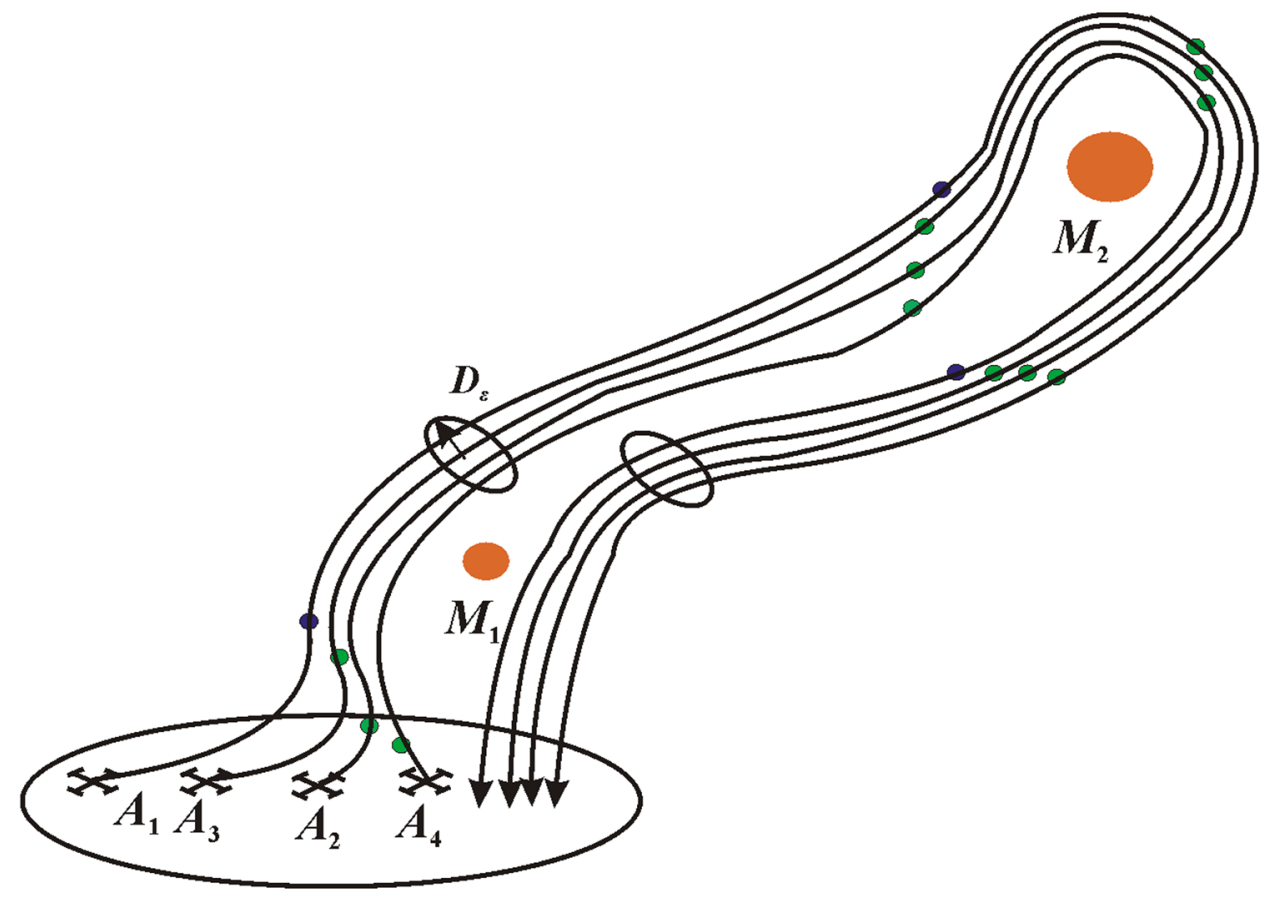

not advisable for the group to maneuver in height, and therefore during the flight, a decision is taken on the variant of the obstacle's bending. The group moves at a constant speed, therefore, the velocities of individual objects of the group are sufficiently close, so the group in the interest of research can be described by some formation called aggregation with a center corresponding to the position of the leader of the group. It is assumed that the agent has the necessary control, navigation, and communication equipment to support the flight. It is also planned to change the number of groups based on the reconciliation protocols.

The obstacle course is on curve trajectory. Thus, the proposed trajectory of motion includes rectilinear and curvilinear parts of the motion. The motion of an agent in a 3-dimensional Euclidean space is considered. The position of the agent is described by some positions pi with coordinates $p_{i}=\left(x_{i}, y_{i}, z_{i}\right)$, and the controlling signal $u_{i}$. Considering the management of agents of a multi-agent system, one can use the dynamic model of a second-order integrator, namely

$\ddot{p}_{i}(t)=k_{i} u_{i}(t)$.

In equation (1), $k_{i}$ is the coefficient describing the reaction of the $i$ th agent, $t \geq 0$ is the time. We also introduce the parameter $D_{\varepsilon}>0$, which determines the size of the multi-agent system, which represents its aggregate for $\forall t \rightarrow \infty$, i.e.

$p_{i} \rightarrow D_{\varepsilon}\left(p_{c}\right)$, 
This suggests that the agent leader is an agent located in the center of the group

$p_{c}(t)=\frac{1}{n} \sum_{i=1}^{n} p_{i}(t)$

and

$D_{\varepsilon}\left(p_{c}\right)=\left\{p \in \Re^{3}: d=\left\|p-p_{c}\right\|<\varepsilon\right\}$.

In (4), $\varepsilon$ acts as a constructive parameter that specifies the safe distance between agents. Thus, formula (4) serves as a criterion for the integrity of the group. It should be noted that the configuration of the group can vary, but the inequality

$D\left(p_{c}\right) \leq D_{\varepsilon}\left(p_{c}\right)$

for the group is always executed.

Let us set the task of synthesizing a control law that ensures the movement of a group on a route with stationary obstacles provided that the group integrity condition (5) is ensured.

\section{THE METHOD OF THE POTENTIAL FIELD}

As already noted, we assume that the agent management system has measurement tools and computing facilities that allow the measurement results to be converted into signals equivalent to attractive and repulsive forces. The force of attraction ensures the integrity of the group, while the forces of repulsion allow avoiding collisions with each other and with obstacles. The emergence of such forces allows us to affirm the existence of potential energies of attraction and repulsion.

By the definition of (Korn \& Korn, 1968), the potential energy of the attractive field is written in accordance with the expression

$$
E_{\text {att }}(\Delta p)=\frac{1}{2} \mu(\Delta p)^{2}
$$

where $\mu>0$ is the scale factor, and $\Delta p_{i}=\left\|p_{i}-p_{c}\right\|$ is the Euclidean distance between the agent and the leader of the group. The form of the homogeneous potential field in the field of the agent-leader is shown in Figure 2.

The effect of this field is most pronounced at the edges and weakened in the center where the leader is located. The force generated by this field is determined by the expression (Jain, 2009)

$u_{a t t}(\Delta p)=-\operatorname{grad} E_{\text {att }}(\Delta p)=-\mu(\Delta p)$. 
Figure 2. Attractive field acting on agent $A_{c}$

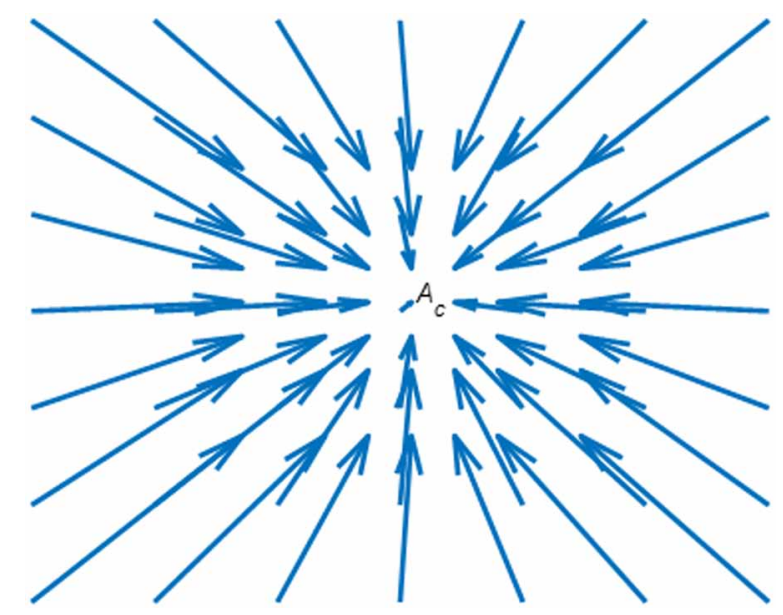

The energy of the repulsive field has the form (Korn \& Korn, 1968)

$$
E_{\text {rep }}\left(\Delta p_{i}\right)=\left\{\begin{array}{l}
\frac{1}{2} \nu\left(\frac{1}{\Delta p_{i}}-\frac{1}{d_{0}}\right)^{2}, \text { if } \Delta p_{i} \leq d_{0} . \\
0, \text { if } \Delta p_{i}>d_{0}
\end{array}\right.
$$

In this expression, $v>0$ is also a scale factor, and $d_{0}>0$ is a certain constant that determines the minimum distance to the nearest neighboring agent. The form of this field is shown in Figure 3, the action of this field is opposite to attracting, so the strongest repulsion occurs at the center and weak at the edges of the aggregation region.

Figure 3. Repulsive field acting on agent $A_{c}$

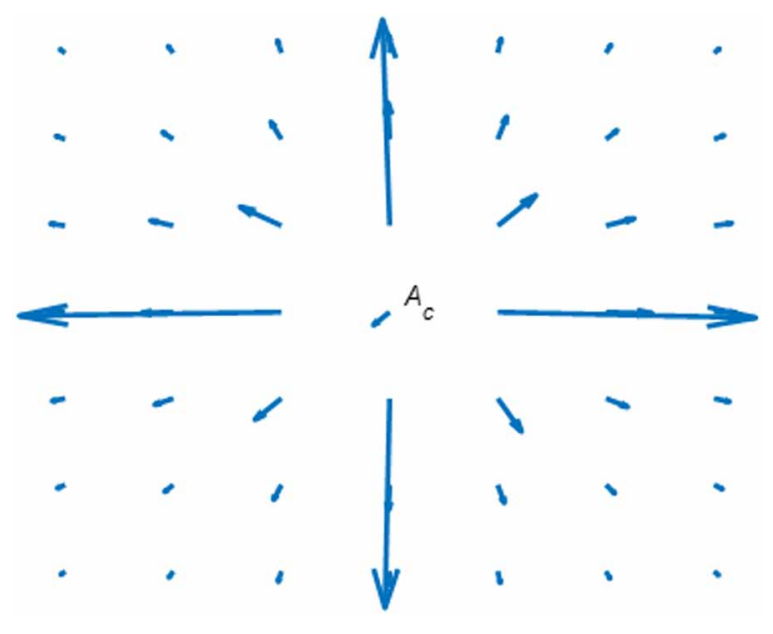


Analogously to (7), we represent the repulsive force by the expression

$$
\begin{aligned}
& u_{\text {rep }}\left(\Delta p_{i}\right)=- \text { grad } E_{\text {rep }}\left(\Delta p_{i}\right) \\
& =\left\{\begin{array}{l}
\nu\left(\frac{1}{\Delta p_{i}}-\frac{1}{d_{0}}\right) \frac{1}{\left(\Delta p_{i}\right)^{2}} \frac{p_{i}-p_{0}}{d_{0}}, \text { if } \Delta p_{i} \leq d_{0}, \\
0, \text { if } \Delta p_{i}>d_{0} .
\end{array}\right.
\end{aligned}
$$

where $p_{i}$ is the closest agent to $p_{0}$ or the obstacles $p_{M}$, and

$d_{0}=\max _{1 \leq i \leq N}\left\|p_{i}-p_{0}\right\|$

Proposition 1. The law governing the $i$ th agent is representing by the following expression

$$
u_{i}^{g}=u^{i}+u_{i}^{a t t}+u_{i}^{r e p},
$$

in which $u_{i}$ has the meaning of the mastering action in aggregation, $u_{i}^{\text {att }}, u_{i}^{\text {rep }}$ is the components of the attractive and repulsive forces, respectively.

Proof. This follows from the accepted idea of the motion of the system in the potential field of attraction and repulsion forces.

The input control $u_{i}^{g}$ of the agent-leader differs from the control law of the $i$ th agent since the control of the latter is building with respect to the leader. Setting the control law of the leader, we will construct an aggregation control trajectory in accordance with the system of equations

$$
\begin{aligned}
& \dot{p}_{1}^{l}=p_{2}^{l}, \\
& \dot{p}_{2}^{l}=k^{l} u^{l},
\end{aligned}
$$

In accordance with (10), the trajectory of the leader starts at the moment $t=0$ from the point $p(0)$ to the point $p\left(t_{k}\right)$. Next, consider the features of aggregation motion on the rectilinear and curvilinear sections of the trajectory.

\section{Control on a Straight Part of a Trajectory}

Considering the assumption that the group moves with approximately equal velocities, i.e. without acceleration, then the system of equations (10) is transformed into the form

$\dot{p}_{1}^{l}=p_{2}^{l}=$ const 
The main task of the agent when moving in a group is to maintain his position, avoiding collisions with neighboring agents or obstacles. For this, the following control law is proposed, representing a smooth function with respect to positions with two neighboring agents

$u^{i}=\left\{\begin{array}{l}-k_{p_{1}}\left(p_{1 i}-p_{1 j}\right)-k_{p_{2}}\left(p_{2 i}-p_{2 j}\right), \text { if } i, j \text { is member }, \\ 0, \text { if } i \text { is not member. }\end{array}\right.$

In (12), $k>0$, positive coefficients that set the value of non-zero deviations $\xi_{p 1} \neq 0, \xi_{p 2} \neq 0$ between the positions of neighboring agents $\left|p_{1 i}-p_{1 j}\right| \leq \xi_{p 1}$ and $\left|p_{2 i}-p_{2 j}\right| \leq \xi_{p 2}$. Assuming the central position of the agent-leader, his position is determined by equations

$p^{l}=\frac{1}{N^{*}} \sum_{i=1}^{N^{*}} p_{i}, \dot{p}^{l}=\frac{1}{N^{*}} \sum_{i=1}^{N^{*}} \dot{p}_{i}$,

where $N^{*}=N-1$ number of neighboring agents in the group. This type of motion is shown in Figure 4 .

Proposition 1. The movement of each agent of a group that consists of $N$ agents is described by the system of differential equations of second order in the form

$\dot{p}_{1}=p_{2}$
$\dot{p}_{2}=\bar{u}=-k_{p_{1}}\left(p_{1 i}-p_{1 j}\right)-k_{p_{2}}\left(p_{2 i}-p_{2 j}\right)$.

where $p=p_{i}-p_{j}$ is the appropriate coordinates of $i$-th and $j$-th agents, $i \neq j, i \leq n$, and $j \leq n$. If $j=l$, is a leader of the group member.

Figure 4. Motion UAVs swarm along line

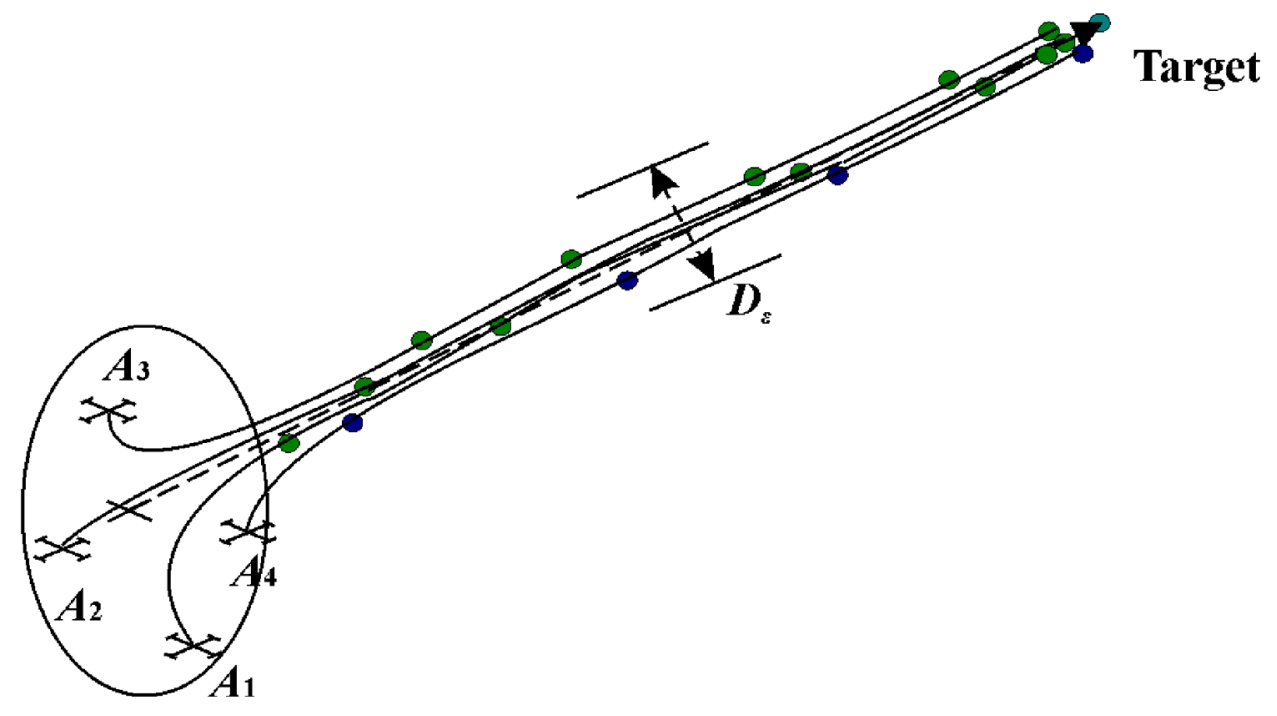


Proof. This statement follows from (9), (11).

Corollary 1. Closed loop dynamic system (14) converges to the equilibrium position

$$
p_{1 i}-p_{1 j} \neq 0, \quad p_{2 i}-p_{2 j} \neq 0
$$

Corollary 2. Agent-members' velocity asymptotically converges to the agent-leader velocity.

Corollary 3. In system (14) coordinates $p_{i}$ is $p_{i} \neq p_{j}$ for any $i \neq j, t \geq 0$.

Corollary 4. If $d=\| p_{i}-p_{j}||<\varepsilon$ is the maximal size between UAVs in a group, the size of formation is $D(p)<D_{\varepsilon}(p)$.

\section{Movement on a Curvilinear Part of the Trajectory}

In this case, the system of equations (11) is transformed into (10), the aggregation moves with acceleration

$$
\begin{aligned}
& \dot{p}_{1}=p_{2}, \\
& \dot{p}_{2}=\bar{u} .
\end{aligned}
$$

In (16), the control signal takes values from the condition $|\bar{u}|<U_{\max }$, the group size becomes larger than on the rectilinear part of the trajectory, but the integrity condition of the group (5) is preserved, as shown in Figure 5.

Proposition 2. Let given factors $k_{y}$ and $k_{p}$ such as $k_{y}>0, k_{p}>0$ is true inequality $0,5 k_{y} / k_{p}<0$, the system (15) has exponential stability.

Figure 5. Motion UAVs along a curve

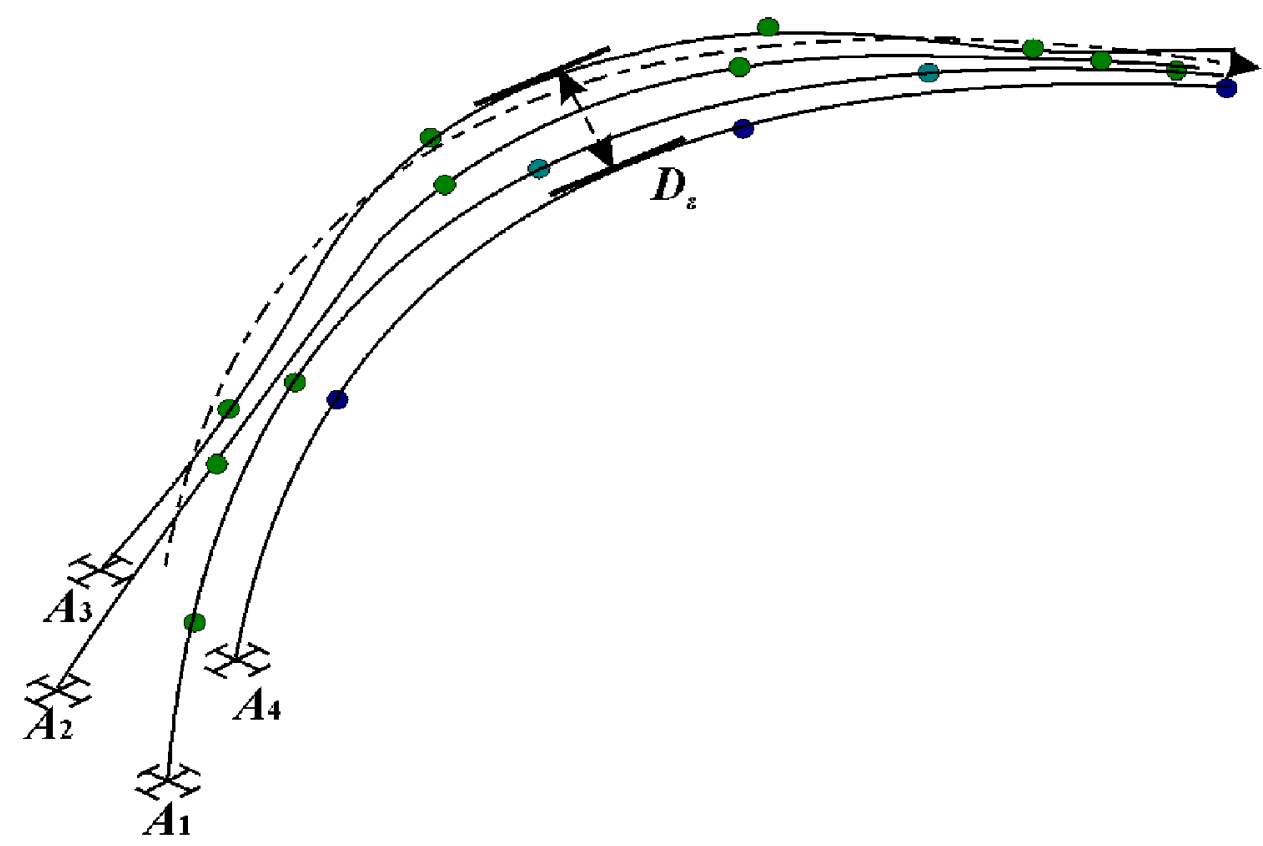


Proof. Let represent the equations system (16) by some differential equation of the second order

$k_{p} \ddot{e}_{p}(t)+k_{y} \dot{e}_{y}(t)+k_{\dot{y}} e_{\dot{y}}(t)=0$

in which $e_{p}=p_{i}-p_{j}, e_{y}=y_{i}-y_{j}, e_{\dot{y}}=\dot{y}_{i}-\dot{y}_{l}$. If the factors $k_{p} \neq 0$, and the $\left(k_{p}, k_{y}, k_{\dot{y}}\right)$ are positive, we can write this equation in the form

$s^{2}(t)+k_{y} / k_{p} s(t)+k_{\dot{y}} / k_{p}=0$.

As known, the exponential stability of (15) is satisfied, if the roots of equation (18) have a negative real part. It is true if the condition $k_{y} /\left(2 k_{p}\right)<0$ is satisfied.

Corollary 1. If factor $k_{y}=0$, we have oscillation motion around the leader with constant amplitude and its frequency is equal to $\sqrt{k_{\dot{y}} / k_{p}}$.

Corollary 2. If condition $k_{y} / \sqrt{4 k_{\dot{y}} k_{p}}>0$ is true, we have asymptotically stability motion.

Corollary 3. If $0<k_{y} / \sqrt{4 k_{\dot{y}} k_{p}}<1$, the agent performs oscillation motion with an error that is decreasing.

Corollary 4. If $k_{y} / \sqrt{4 k_{\dot{y}} k_{p}}>1$, the agent performs a periodic motion.

\section{Features of Motion in Conditions of Obstacles}

Movement in obstacle conditions is the most complex movement since it requires the inclusion of additional components in the law of control, based on the forces of attraction and repulsion. The motion occurs along the curvilinear part of the trajectory, therefore, to describe the dynamics of the system, a system of equations (16) with a control law of the form

$$
u_{i}=\left\{\begin{array}{l}
u_{\text {rep } p i}+u_{\text {rep } p_{1 i}}+u_{\text {rep } p_{2 i}}, \\
\text { if }(d-2 r) \leq d_{0}, \quad p_{1 i}>0, \quad p_{2 i}>0 \\
u_{\text {rep } p_{i}}+u_{\text {rep } p_{1 i}}, \\
\text { if }(d-2 r) \leq d_{0}, \quad p_{1 i}>0, \quad p_{2 i} \leq 0, \\
0, \text { if }(d-2 r)>d_{0}, \text { or } p_{2 i} \leq 0,
\end{array}\right.
$$

in which

$$
\begin{aligned}
& u_{r e p p_{i}}=k_{1}\left(\frac{1}{d-2 r}-\frac{1}{d_{0}}\right), \\
& u_{r e p p_{1 i}}=k_{2} p_{1 i o b s},
\end{aligned}
$$


$u_{\text {rep } p_{2 i}}=k_{3} p_{2 i o b s}$,

parameter $r$ sets the maximum size of the agent in the control plane,

$p_{1 i o b s}=p_{1 i}^{T} n_{\text {aobs }}$,

and

$p_{2 i o b s}=p_{2 i o b s}{ }^{T} n_{a o b s}$,

where $n_{\text {aobs }}$ is a unit vector pointing from $i$-th agent to obstacle.

The parameter $p_{1 i o b s}$ sets the direction of the agent's movement to the obstacle, so if $p_{1 i o b s}>0$, then the agent moves towards the obstacle, otherwise, i.e. if $p_{1 i o b s} \leq 0$ the agent moves from the obstacle. One of the options for overcoming obstacles by a group of 4 agents is shown in Figure 6 .

Since the obstacles are arranged so that the group is able to overcome this type only if the configuration is changed, i.e. when reconfiguration. In this case, the reconfiguration occurs with the fulfillment of the condition (13) for the agent leader.

\section{MODEL UAV}

\section{Main Concepts}

Under the conditions of the problem under consideration, each agent of the group is an unmanned aerial vehicle (Bouabdallah \& Siegwart, 2005), the traction force of which is created by 4 propellers controlled by motors via autonomous control channels. Propellers are installed in the same plane at equal distances

Figure 6. Motion N=4 UAVs in obstacles

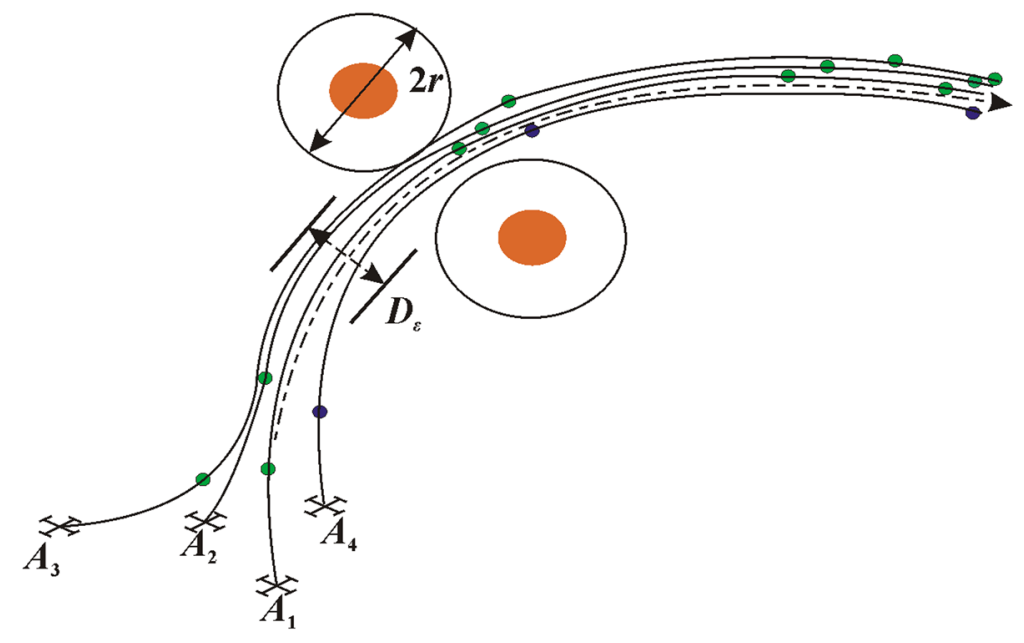


from the center of mass and on mutually perpendicular axes. Then scheme for creating control actions is similar to a helicopter (Olfati-Saber, 2001), which allows vertical takeoff and landing, as well as easy to change the direction of the vehicle, including hanging. In this case, the direction of rotation of a pair of propellers located along one axis is set opposite to the direction of rotation of the second pair located on the perpendicular axis, Figure 7.

Then, to represent the UAV, we use the equations of motion of the helicopter (Olfati-Saber, 2001; Bouabdallah \& Siegwart, 2005)

$$
\left\{\begin{array}{l}
\dot{x}=v, \\
m \dot{v}=R F_{b}, \\
\dot{R}=R \hat{\omega}, \\
J \dot{\omega}=-\omega \times J \omega+\tau_{b} .
\end{array}\right.
$$

In equation system (25) $\mathrm{x}$ is the position vector, and $\mathrm{v}$ is the velocity vector of the UAV center of mass relative to the origin, $\mathrm{m}$ is the mass of the apparatus, $J$ is the inertia matrix, $J \in \mathfrak{R}^{3 \times 3}$, it is given by the formula

$$
J=\left|\begin{array}{ccc}
I_{x x} & I_{x y} & I_{x z} \\
I_{y x} & I_{y y} & I_{y z} \\
I_{z x} & I_{z y} & I_{z z}
\end{array}\right|=-\int_{V} \rho(r) \hat{r}^{2} d V
$$

and $R$ is the rotation matrix of the $\mathrm{UAV}$ in the frame $O$, it has the form

$$
R=\exp \left(\psi e_{3}\right) \exp \left(\theta e_{2}\right) \exp \left(\varphi e_{1}\right)
$$

where $e_{i} \in \Re^{3}$ is the unit vector basis for the $X Y Z$ coordinate system, and $\varphi, \theta, \psi$ are the angles associated with rotations about the $x, y, z$ axes, in the Eulerian coordinate system these angles are called roll, pitch,

Figure 7. The configuration of 4-motor UAV with fixed body frame and inertial frame $O$

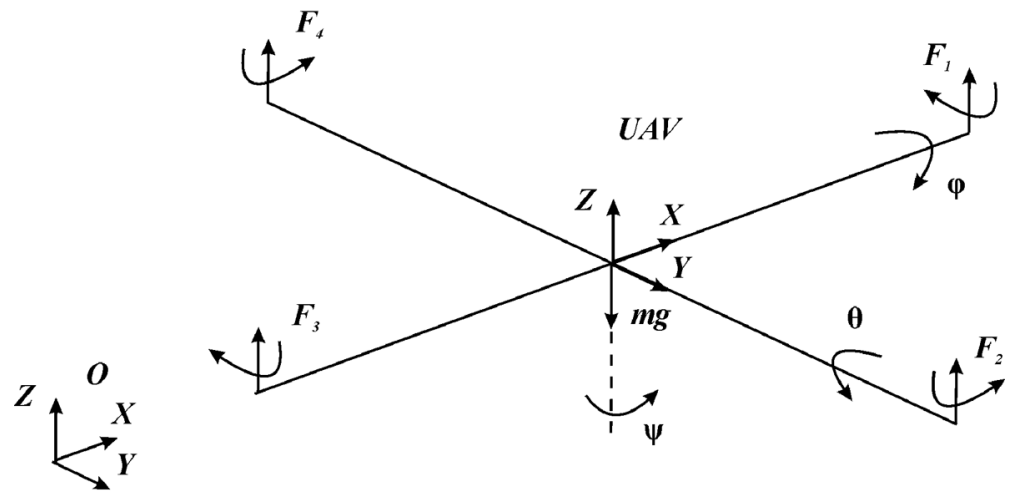


and yaw, respectively. $\omega$ denotes the angular velocity vector, $\omega=\left(\omega_{1}, \omega_{2}, \omega_{3}\right)^{\mathrm{T}}$, the symbol $(\times)$ denotes the vector product. $F_{b}, \tau$ are the forces and moments that are written in the form

$$
\begin{aligned}
& F_{b}=-m g R^{T} e_{3}+T e_{T}, \\
& \tau_{b}=Q(T) e_{T}+D \tau,
\end{aligned}
$$

$T$ is the value characterizing the main thrust of the apparatus, and $e_{T}$ is the unit vector along the path of the aircraft, which has a small inclination and angles of rotation relative to the central axis,

$$
e_{T}(a, b)=\left|\begin{array}{c}
\sin (a) \\
-\cos (a) \sin (b) \\
\cos (a) \cos (b)
\end{array}\right| \text {. }
$$

In equation (28), $\tau=\left(b_{T}, a_{T}\right)$, and $D=\operatorname{diag}\left(l_{b}, l_{a}\right)$ is the matrix whose elements of the main diagonal are positive numbers. Thrust may vary according to law

$$
Q(T)=Q_{0}+Q_{1} T^{1.5}
$$

with values $Q_{0}>0, Q_{1}>0$.

We will assume small values $a, b$, and $D_{\tau}=u \in \mathfrak{R}^{3}$ and use the approximation $Q(T) e_{T} \approx Q(T) e_{3}$ then (28) is written in the form

$$
\begin{aligned}
& F_{b}=-m g R^{T} e_{3}+T e_{3}, \\
& \tau_{b}=Q(T) e_{3}+\tau .
\end{aligned}
$$

Further simplification is connected with the assumption small values $(a, b)$, and $e_{T}=\mathrm{e}_{3}$, equation (29) can write in the form

$$
e_{T} \approx e_{3}+\left|\begin{array}{c}
a \\
-b \\
0
\end{array}\right|=\left|\begin{array}{c}
a \\
-b \\
1
\end{array}\right| .
$$

By setting the values of $a, b$, essentially small, and giving the values of $T, \tau$ one can obtain the system of equations (25) look as follows 


$$
\left\{\begin{array}{l}
\dot{x}=v, \\
m \dot{v}=-m g e_{3}+\left(R e_{3}\right) T, \\
\dot{R}=R \hat{\omega} \\
J \dot{\omega}=-\omega \times J \omega+Q(T) e_{3}+\tau .
\end{array}\right.
$$

Now present $R e_{3}$ on the product of the unit normal vector and matrix $R$, then matrix $R$ we can represent in the form

$$
R=\left|\begin{array}{ccc}
C_{\theta} C_{\psi} & S_{\phi} S_{\theta} C_{\psi}-C_{\phi} S_{\psi} & C_{\phi} S_{\theta} C_{\psi}+S_{\phi} S_{\psi} \\
C_{\theta} S_{\psi} & S_{\phi} S_{\theta} S_{\psi}+C_{\phi} C_{\psi} & C_{\phi} S_{\theta} S_{\psi}-S_{\phi} C_{\psi} \\
-S_{\theta} & S_{\phi} C_{\theta} & C_{\phi} C_{\theta}
\end{array}\right|,
$$

where $C(\cdot)=\cos (\cdot), S(\cdot)=\sin (\cdot)$. The dynamics of the agent in the system (33) is given by the variable $T$, which should be associated with the master effect. We replace it by the letter $U$, which is usually used in the theory of control systems, then we represent the second equation in (33) in the form

$m \dot{v}=-m g e_{3}+\left(R e_{3}\right) U$,

where

$U=F_{1}+F_{2}+F_{3}+F_{4}$

(see Figure 7), and

$$
F_{i}=\alpha_{i} \omega_{i}^{2}
$$

Here $\alpha_{i}>0$ is the some constant, and $\omega_{i}$ is angular velocity $i$-th motor $(i=1, \ldots, 4)$.

We are going to consider only the longitudinal motion on the axis $Y$, then the vector $\tau$ will have the form

$\tau=\left(\tau_{\phi}, \tau_{\theta}, \tau_{\psi}\right)^{T}$,

with the components

$$
\begin{gathered}
\tau_{\phi}=l\left(F_{4}-F_{2}\right), \\
\tau_{\theta}=l\left(F_{3}-F_{1}\right),
\end{gathered}
$$


$\tau_{\psi}=l\left(F_{4}+F_{2}-F_{3}-F_{1}\right)$.

In equations (39) - (41), the variable $l$ denotes the distance from the center of mass of the apparatus to the motor.

We will assume that the input signal in (25) is used to control the quadrotor when flying to a given point. In this case, the yaw pull component $\tau \psi$ is close to zero, and the pitches thrust $\tau_{\theta}$ and roll $\tau_{\varphi}$ provide stabilization of the angular position. This makes it possible to simplify the right-hand side in (25) for rotational motion and to replace the input signals for individual moments.

Then (25) look like follow

$\ddot{\psi}=\bar{\tau}_{\psi},$.

$\ddot{\theta}=\bar{\tau}_{\theta}$,

$\ddot{\phi}=\bar{\tau}_{\phi}$

$m \ddot{x}=\left(C_{\phi} S_{\theta} C \psi+S_{\phi} S \psi\right) U$,

$m \ddot{y}=\left(C_{\phi} S_{\theta} S_{\psi}-S_{\phi} S \psi\right) U$

$m \ddot{z}=-m g+C_{\phi} C_{\theta} U$.

The equations (43) - (47) coincide with the equations (6) from (Ren et al, 2005) if in right parts (6) from (Kucherov, 2016) enter variables $\bar{\tau}_{\psi}, \bar{\tau}_{\theta}, \bar{\tau}_{\phi}$, and we can obtain the equations (18) - (23) from (Bouabdallah \& Siegwart, 2005) if we take $\psi=0$, and

$U=b\left(F_{1}+F_{2}+F_{3}+F_{4}\right)$.

\section{Control Law}

In the interests of modeling, equations (42) - (43) can be represented in the form of differential equations with respect to angular velocities $\dot{\varphi}, \dot{\theta}, \dot{\psi}$, taking into account the moments of inertia $I_{1}, I_{2}, I_{3}(26)$ by axes $X, Y$, and $Z$ of the apparatus 
$I_{1} \varphi(t)=\left(I_{2}-I_{3}\right) \dot{\theta}(t) \dot{\psi}(t)$

$I_{2} \theta(t)=\left(I_{3}-I_{1}\right) \dot{\psi}(t) \dot{\varphi}(t)$

$I_{3} \psi(t)=\left(I_{1}-I_{2}\right) \dot{\varphi}(t) \dot{\theta}(t)$

Then a model of quadrotor is presented by following equations system

$$
\left\{\begin{array}{l}
\ddot{\varphi}=\dot{\theta} \dot{\psi}\left(\frac{I_{y}-I_{x}}{I_{x}}\right)-\frac{J_{r}}{I_{x}} \dot{\theta} w+\frac{l}{I_{x}} U_{2}, \\
\ddot{\theta}=\dot{\varphi} \dot{\psi}\left(\frac{I_{z}-I_{x}}{I_{y}}\right)-\frac{J_{r}}{I_{y}} \dot{\varphi} w+\frac{l}{I_{y}} U_{3}, \\
\ddot{\psi}=\dot{\varphi} \dot{\theta}\left(\frac{I_{x}-I_{y}}{I_{z}}\right)+\frac{1}{I_{z}} U_{4}, \\
\ddot{z}=-g+(\cos \varphi \cos \theta) k U_{1}, \\
\ddot{x}=(\cos \varphi \cos \theta \cos \psi+\sin \varphi \sin \psi) k U_{1}, \\
\ddot{y}=(\cos \varphi \cos \theta \cos \psi+\sin \varphi \cos \psi) k U_{1}
\end{array}\right.
$$

Here $(\phi, \theta, \psi)$ is the roll, pitch and yaw angles, $I_{x, y, z}$ is the inertia UAV, $J_{r}$ is the inertia rotor, $l$ is the lever in coordinate system with the Quadrotor, $k=1 / m, m$ is the mass UAV, $w$ is the rotor speed, and $U_{1}, U_{2}, U_{3}, U_{4}$, are calculated as

$$
\left\{\begin{array}{l}
w=w_{2}+w_{4}-w_{1}-w_{3} \\
U_{1}=b\left(w_{1}^{2}+w_{2}^{2}+w_{3}^{2}+w_{4}^{2}\right) \\
U_{2}=b\left(w_{4}^{2}-w_{2}^{2}\right) \\
U_{3}=b\left(w_{3}^{2}-w_{1}^{2}\right) \\
U_{4}=d\left(w_{2}^{2}+w_{4}^{2}-w_{1}^{2}-w_{3}^{2}\right)
\end{array}\right.
$$

where $\mathrm{b}$ is the thrust factor, and $\mathrm{d}$ is the drag factor.

For simulation, the model (51) is transforming to a space-state form $\dot{X}=f(X, U)$, where $X \in \mathfrak{R}^{12}$ is the state vector with the coordinates 
$\dot{X}=\left\{\begin{array}{l}x_{2}, \\ x_{4} x_{6} a_{1}+x_{4} a_{2} w+b_{1} U_{2}, \\ x_{4}, \\ x_{2} x_{6} a_{3}+x_{2} a_{4} w+b_{2} U_{3}, \\ x_{6}, \\ x_{4} x_{2} a_{5}+b_{3} U_{4}, \\ x_{8}, \\ -g+\left(\cos x_{1} \cos x_{3}\right) k U_{1}, \\ x_{10}, \\ u_{x} k U_{1}, \\ x_{12}, \\ u_{y} k U_{1},\end{array}\right.$

where

$$
\begin{aligned}
& x_{1}=\varphi, x_{2}=\dot{x}_{1}=\dot{\varphi}, x_{3}=\theta, x_{4}=\dot{x}_{3}=\dot{\theta}, \\
& x_{5}=\psi, x_{6}=\dot{x}_{5}=\dot{\psi}, x_{7}=z, x_{8}=\dot{x}_{8}=\dot{z} \\
& x_{9}=x, x_{10}=\dot{x}_{9}=\dot{x}, x_{11}=y, x_{12}=\dot{x}_{11}=\dot{y} .
\end{aligned}
$$

$a_{1}=\frac{\left(I_{y}-I_{z}\right)}{I_{x}}, a_{2}=-\frac{J_{r}}{I_{x}}, a_{3}=\frac{\left(I_{z}-I_{x}\right)}{I_{y}}, a_{4}=\frac{J_{r}}{I_{y}}$,

$a_{5}=\frac{\left(I_{x}-I_{y}\right)}{I_{z}}, b_{1}=\frac{l}{I_{x}}, b_{2}=\frac{l}{I_{y}}, b_{3}=\frac{l}{I_{z}}$,

$u_{x}=\cos x_{1} \sin x_{3} \cos x_{5}+\sin x_{1} \sin x_{5}$,

$u_{y}=\cos x_{1} \sin x_{3} \cos x_{5}-\sin x_{1} \sin x_{5}$.

The translation model dynamic (51) can be represented in form three-dimensional double-integrators

$$
\begin{aligned}
& \dot{\eta}=p, \\
& p=U,
\end{aligned}
$$

then 


$$
\begin{aligned}
& \dot{x}=V_{x}, \quad \dot{V}_{x}=\left(C_{\phi} S_{\theta} C_{\psi}+S_{\phi} S_{\psi}\right) U / m, \\
& \dot{y}=V_{y}, \quad \dot{V}_{y}=\left(C_{\phi} S_{\theta} C_{\psi}-S_{\phi} S_{\psi}\right) U / m, \\
& \dot{z}=V_{z}, \quad \dot{V}_{z}=-g+C_{\phi} C_{\theta} U / m .
\end{aligned}
$$

The control law of the $i$ th agent is synthesized on the basis of the coordinate information regarding the position and speed of the leader, it does not coincide with the management of the leader and other agents not belonging to the group

$U_{i}=\left\{\begin{array}{l}-k_{y}\left(y_{i}-y^{l}\right)-k_{x}\left(x_{i}-x^{l}\right), \text { if } i \text { is agent }- \text { member }, \\ 0, \text { else }\end{array}\right.$

Factor $k>0$ set safe distance between agents. In contrast to [39], which used to create a sliding mode for control, the control actions $\left(U_{1}, U_{2}, U_{3}, U_{4}, u_{x}, u_{y}\right)$ of each agent are smooth functions of time $t$.

\section{SIMULATION}

In this section, we consider the motion of a group of quadrotors moving in the potential field of the forces of attraction and repulsion towards the target along a route on which there are obstacles.

We will assume that the group consists of four agents, one of them is the leader. The route of agents is given by two points: the initial and final points. The stratum position of the quadrotors $p_{A 1}=(101$, $31,0)^{\mathrm{T}}, p_{\mathrm{A} 2}=(76,22,0)^{\mathrm{T}}, p_{\mathrm{A} 3}=(131,51,0)^{\mathrm{T}}, p_{\mathrm{A} 41}=(120,72,0)^{\mathrm{T}}$, endpoint of the trajectory $p_{t}=(610$, $275,0)^{\mathrm{T}}$. Coordinates of the stationary obstacles located on the route $p_{O 1}=(265,133)^{\mathrm{T}}, p_{O 2}=(221,211)$ ${ }^{\mathrm{T}}$ and $D_{\varepsilon}\left(p_{A}\right) \leq 45$ are also given. It is assumed that the height obstacles are higher than the altitude of the quadrotors, which leads to maneuvering around the obstacles. The height coordinate is taken equal to $z=15$. The simulation of the movement of a group of agents is shown in Figure 8 .

It can be seen from Figure 8 that a group of agents when passing a route, maneuvers around the obstacle and continues to move to the target along the planned route. If in order to overcome obstacles, it is required to change the size of its aggregation, the group increases distances, and the agent aggregation form remains unchanged if the route has no obstacles.

Figure 9 shows an example of the reconfiguration of a UAV group in the presence of obstacles, the placement of which does not allow the use of an obstacle avoidance maneuver. At the same time, the group is lined up if obstacles along the route are on the way. In this case, the UAV tactics are similar to the column and the UAVs follow in a strictly defined order: A4 $\rightarrow$ A3 $\rightarrow$ A2 $\rightarrow$ A1, where A1 is the first and A4 is the last one. 
Figure 8. Bypass movement of agent-leader and agent-members to the target

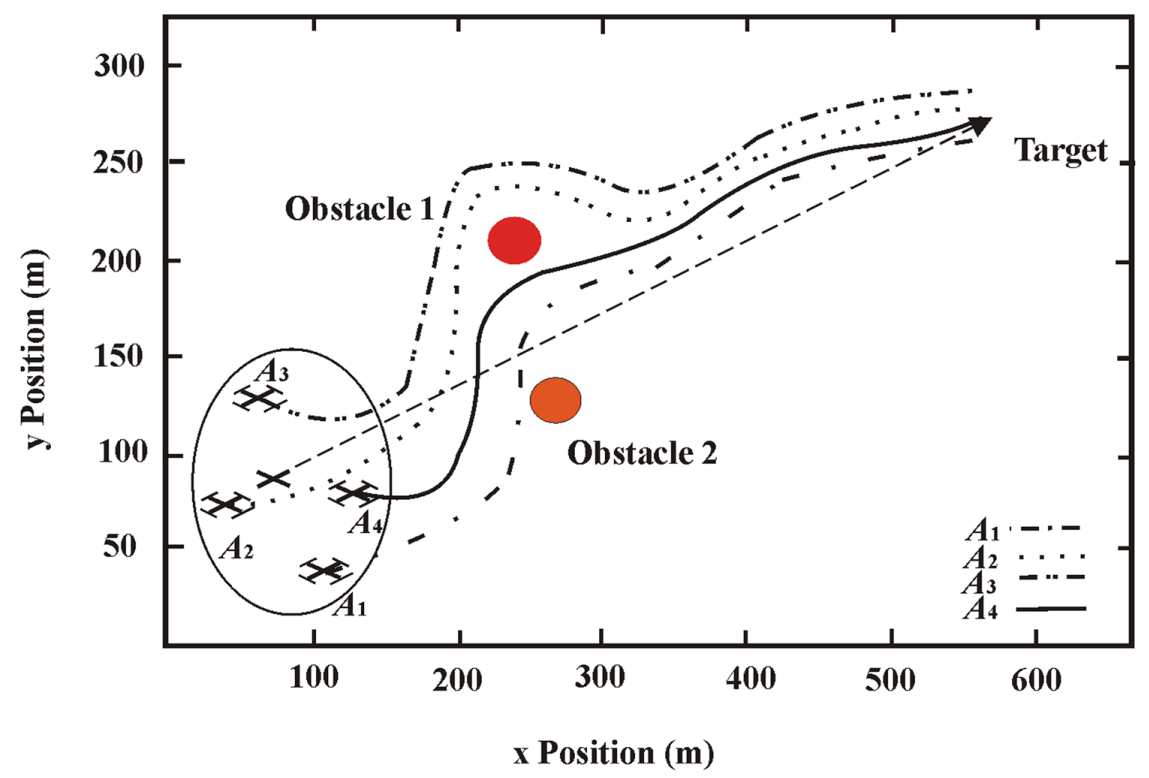

Figure 9. Group of UAVs overcomes side-by-side obstacles by line type motion

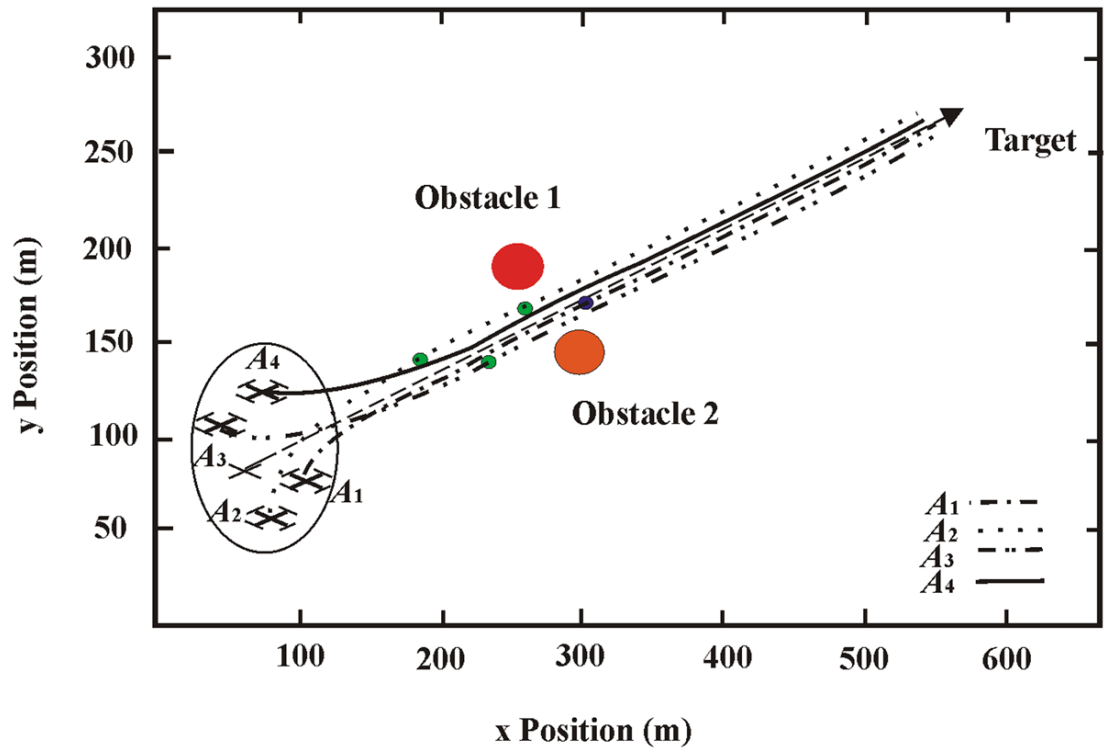

\section{CONCLUSION}

The article deals with the problem of synthesis of a smooth motion control law by a group consisting of a limited number of agents following a given route on which obstacles to movement are possible. Obstacles can be overcome in two possible ways: namely to bypass the obstacle without changing the traffic configuration, and the other is based on the reconfiguration of the group, preserving the integrity 
of the group. In order to avoid the risk of collision of group agents with obstacles and with each other, additional components associated with repulsion forces are introduced into the control law, which does not allow agents to approach closer than a certain predetermined distance and to be removed in order to keep the specified configuration. The need to overcome obstacles leads to a change in the initial configuration of the group.

Based on the idea of the motion of a group of agents in the potential field of attraction and repulsion, the control law of the movement of a group is synthesized, and an algorithm was developed to simulate the agent system under investigation. The properties of the algorithm were studied, which makes it possible to determine certain parameters of the stable motion of a group under given conditions. The presented examples allow talking the efficiency of the algorithm in the considered flying situations. Modeling made it possible to provide the aperiodic character of the trajectory of agents in possible sections of rectilinear and curvilinear motion. The success of the simulation also depended on the choice of parameters, like $\mu$ and $\mathrm{v}$ potential functions.

Since the motion along the curve of the trajectory is more general, during its investigation it was found necessary to increase the amplitude of the control signal in order to exclude the increase in the time of the motion. However, the operation with the maximum amplitude control signal can cause "sliding mode" when the input signal is frequently switched. Investigation of the agent system in a sliding mode is one of the future topics of our research.

\section{REFERENCES}

Amelin, K., Amelina, N., Granichin, O., Granichina, O., \& Andrievsky, B. (2013). Randomized Algorithm for UAVs Group Flight Optimization. In 11th IFAC International Workshop on Adaptation and Learning in Control and Signal Processing. University of Caen Basse-Normandie. 10.3182/201307033-FR-4038.00130

Baolin, F., Lu, L., Lu, X., \& Zhu, K. (2017). An Optimized Cooperative Control Algorithm of Multi-Agent Array Based on the Skeletal Mechanism of Chrysopelea. Proceedings of the 2017 IEEE International Conference on Robotics and Biomimetics, 2003 - 2008.

Bouabdallah, S., \& Siegwart, R. (2005). Backstepping and Sliding-mode Techniques Applied to an Indoor Micro Quadrotor. IEEE International Conference on Robotics and Automation, 2259 - 2264. 10.1109/ROBOT.2005.1570447

Ferber, J., Gutknecht, O., \& Michel, F. (2004). From Agents to Organizations: An Organizational View of Multi-Agent Systems. LNCS, 2935, 214-230.

Filaretov, V., Yukhimets, D., \& Bidyadhar, S. (2017). Information-Control System for Cooperative Control of a Mobile Robots Group. International Conference on Industrial Engineering, Applications and Manufacturing (ICIEAM), 1 - 6. 10.1109/ICIEAM.2017.8076169

Flemisch, F., Canpolat, Y., Altendorf, E., Weßel, G., Flemisch, F., ... Schutte, P. (2017). Shared and cooperative control of ground and air vehicles: Introduction and General overview. IEEE International Conference on Systems, Man, and Cybernetics (SMC), 858 - 863. 10.1109/SMC.2017.8122717 
Franchi, A., Giordano, P. R., Secchi, C., Son, H. I., \& Bulthoff, H. H. (2011). A Passivity-Based Decentralized Approach for the Bilateral Teleoperation of a Group of UAVs with Switching Topology. IEEE International Conference on Robotics and Automation, 898 - 905. 10.1109/ICRA.2011.5980368

Han, L., Cheng, X., Xu, W., \& Tan, G. (2017). Reconfigurable Wireless Control System for a Dual-arm Cooperative Robotic System. Proceedings of the 2017 IEEE International Conference on Robotics and Biomimetics, 202-207. Retrieved October 09, 2018, from https://ieeexplore.ieee.org/document/8324418/

Hao, Y., Pan, S., Qiao, Y., \& Cheng, D. (2018). Cooperative Control via Congestion Game Approach. IEEETransactions on Automatic Control, 9. Retrieved from https://ieeexplore.ieee.org/document/8334284/

Hoek, W., \& Wooldridge, M. (2008). Multi-Agent Systems. In Handbook of Knowledge Representation (pp. 1-41). Amsterdam: Elsevier. doi:10.1016/S1574-6526(07)03024-6

Hu, J., \& Hong, Y. (2007). Leader-following Coordination of Multi-Agent Systems with Coupling Time Delays. Physica A, 374(2), 853-863. doi:10.1016/j.physa.2006.08.015

Jain, M. C. (2009). Textbook of Engineering Physics (Part I). Delhi, India: PHI Learning Private Ltd.

Kala, R., \& Warwick, K. (2012). Planning Autonomous Vehicles in the Absence of speed Lanes using Lateral Potentials. Proceedings of the 2012 IEEE Intelligent Vehicles Symposium, 597-602. 10.1109/ IVS.2012.6232148

Koo, T. J., \& Shahruz, S. M. (2001). Formation of a Group of Unmanned Aerial Vehicles (UAVs). Proceedings of the American Control Conference Arlington, 69-74. Retrieved October 09, 2018, from https://ieeexplore.ieee.org/document/945515/

Korn, G. A., \& Korn, T. M. (1968). Mathematical Handbook for scientist and engineers. McGraw-Hill Company.

Kozlovskyy, V., Parkhomey, I., Odarchenko, R., Gnatyuk, S., \& Zhmurko, T. (2016). Method for UAV trajectory parameters estimation using additional radar data. Proceedings IEEE 4th International Conference Methods and Systems of Navigation and Motion Control (MSNMC), 39 - 42. 10.1109/ MSNMC.2016.7783101

Kucherov, D. P. (2014). Agent-based approach to the problem movements by the route, Visnik of the Volodymyr Dahl East Urk. National University, 4(211), 168-171.

Kucherov, D. P., \& Kozub, A. N. (2015). Control System Objects with Multiple Stream of Information. Proceedings IEEE 3rd International Conference "Actual Problems of Unmanned Aerial Vehicles Developments (APUAVD)”, 290 - 293. 10.1109/APUAVD.2015.7346623

Kucherov, D. P., Kozub, A. N., \& Kostyna, O. N. (2016). Group Behavior of UAVs in Obstacles Presence. Proceedings IEEE 4th International Conference "Methods and Systems of Navigation and Motion Control” (MSNMC), 51 - 54. 10.1109/MSNMC.2016.7783104

Kulik, A. (2016). Intelligent Transport System in Aerospace Engineering, in Intelligent transportation systems-problems and perspectives. Springer International Publishing. Retrieved October 09, 2018, from http://www.springer.com/us/book/9783319191492 
Li, W. (2018, March). The Constrained Rayleigh Quotient with a General Orthogonality Constraint and an Eigen-Balanced Laplacian Matrix: The Greatest Lower Bound and Applications in The Greatest Lower Bound and Applications in Cooperative Control Problems. IEEE Transactions on Automatic Control, 12. Retrieved from https://ieeexplore.ieee.org/abstract/document/8314760/

Liu, L., Liang, X., Zhu, C., \& He, L. (2017). Distributed Cooperative Control for UAV Swarm Formation Reconfiguration Based on Consensus Theory. 2nd International Conference on Robotics and Automation Engineering, 264-268. Retrieved October 09, 2018, from https://ieeexplore.ieee.org/document/8291392/

Ma, C.-Q., \& Zhang, J.-F. (2010). Necessary and Sufficient Conditions for Consensusability of Linear Multi-Agent Systems. IEEE Transactions on Automatic Control, 55(5), 1263-1268. doi:10.1109/ TAC.2010.2042764

Murray, R.M. (2007). Recent Research in Cooperative Control of Multi-Vehicle Systems. ASME Journal of Dynamic Systems, Measurement, and Control, 1 - 27.

Ni, W., \& Cheng, D. (2010). Leader-following consensus of multi-agent systems under fixed and switching topologies. Systems \& Control Letters, 59(3-4), 209-217. doi:10.1016/j.sysconle.2010.01.006

Ögren, P., Fiorelli, E., \& Leonard, N. E. (2004). Cooperative Control of Mobile Sensor Networks: Adaptive Gradient Climbing in a Distributed Environment. IEEE Transactions on Automatic Control, 49(8), 1292-1302. doi:10.1109/TAC.2004.832203

Olfati-Saber, R. (2001). Nonlinear control of underactuated mechanical systems with application to robotics and aerospace vehicles (Ph.D. thesis). MIT.

Olfati-Saber, R., Dunbar, W. B., \& Murray, R. M. (2003). Cooperative Control of Multi-Vehicle Systems using Cost Graphs and Optimization. Proceedings of the 2003 American Control Conference, 1 - 15. Retrieved October 09, 2018, from https://ieeexplore.ieee.org/document/1243403/

Olfati-Saber, R., Fax, J. A., \& Murray, R. M. (2007). Consensus and Cooperation in Networked MultiAgent Systems. Proceedings of the IEEE, 95(1), 215-233. doi:10.1109/JPROC.2006.887293

Pipattanasomporn, M., Feroze, H., \& Rahman, S. (2009). Multi-Agent Systems in a Distributed Smart Grid: Design and Implementation. Proceeding IEEE PES 2009 Power Systems Conference and Exposition (PSCE'09), 1 - 8. 10.1109/PSCE.2009.4840087

Ren, W., Beard, R. W., \& Atkins, E. M. (2005). A Survey of Consensus Problems in Multi-Agent Coordination. American Control Conference, 1859 - 1864.

Rezaee, H., \& Abdollah, F. (2016). Consensus Problem in General Linear Multi-Agent Systems Under Stochastic Topologies. IFAC-PapersOnLine, 49(13), 13-18. doi:10.1016/j.ifacol.2016.07.919

Shmelova, T., Sikirda, Y., Zemlyanskiy, A., Lazoprenko, V., \& Danilenko, O. (2016). Artificial neural network for air traffic controller's pre-simulator training. Proceedings of the National Aviation University, (3), 13 - 23. 10.18372/2306-1472.68.10905

Xiao, F., Wang, L., Chen, J., \& Gao, Y. (2009). Finite-time formation control for multi-agent systems. Automatica, 45(11), 2605-2611. doi:10.1016/j.automatica.2009.07.012 
Xie, Y., Shi, Z., Sun, C., Deng, Y., \& Wu, X. (2017). The Integration of Adaptive Cooperative Control for Position and Attitude of Distributed Spacecraft. Proceedings of the 36th Chinese Control Conference, 3409 - 3413. 10.23919/ChiCC.2017.8027886

Yu, X., Guo, Ge., \& Gong, G. (2017). Cooperative control for a platoon of vehicles with leader-following communication strategy. Chinese Automation Congress (CAC), 6721 - 6726. Retrieved October 09, 2018, from https://ieeexplore.iee.org/document/8243988/

Yun, L., \& Jian, Z. (2018). Design and Implementation of Cooperative Turning Control for the Towing System of Unpowered Facilities. IEEE Access: Practical Innovations, Open Solutions, 6, 18713-18722. doi:10.1109/ACCESS.2018.2819692

Zhang, H., \& Chen, J. (2014). Bipartite Consensus of Linear Multi-Agent Systems over Signed Digraphs: An Output Feedback Control Approach. Proceedings of the 19th World Congress the International Federation of Automatic Control, 4681-4686. 10.3182/20140824-6-ZA-1003.00608

Zuo, Z., Han, Q.-L., Ning, B., Ge, X., \& Zhang, X.-M. (2018). An Overview of Recent Advances in Fixed-Time Cooperative Control of Multi-Agent Systems. IEEE Transactions on Industrial Informatics, 14(6), 2322-2334. doi:10.1109/TII.2018.2817248

\section{ADDITIONAL READING}

Cooper, R. B. (1981). Introduction to Queueing Theory. Amsterdam: Elsevier North Holland.

Cormen, T. H., Leiserson, C. E., Rivest, R. L., \& Stein, C. (2002). Introduction to Algorithms. McGrawHill Book Company.

Marghitu, D. B. (2001). Mechanical Engineer's Handbook. Orlando, FL: Academic Press.

Rodic, A. D. (2009). Automation \& Control - Theory and Practice. Zagreb, Croatia: In-Tech.

Taha, A. H. (2003). Operations Research: An Introduction. London: Pearson Education Inc.

\section{KEY TERMS AND DEFINITIONS}

Agent: Some entity, a system that possesses properties, in addition to the properties of the object to exist and unite, necessary for interaction with the external environment.

Aggregate: System, the combination of something combined to fulfill a specific goal.

Attractive Field: This is a potential vector field around each body whose action opposite to repulsive field.

Control Law: The mathematical form of transformations of driving influences, disturbances, effects of feedbacks that determine the control actions.

Group: A set of related and interacting elements.

Movement: This called the change in position of a body in space relative to other bodies over time. In this case, the bodies interact according to the laws of mechanics. 
Synthesis of the Laws of Motion Control of a UAV Group With Natural Obstacles

Potential Field: A vector field that can be represented as the gradient of some scalar coordinate function.

Repulsive Field: This is a potential vector field around each body whose strength is inversely proportional to its distance.

UAV: Unmanned aerial vehicle or drone is an aircraft without crew on board. 